\title{
Performance of Rotated Lattice Constellations in Fading Channels
}

\author{
Albert Guillén i Fàbregas \\ Institute for Telecommunications Research \\ University of South Australia \\ Mawson Lakes SA 5095, Australia \\ albert.guillen@unisa.edu.au
}

\author{
Emanuele Viterbo \\ Dipartimento di Elettronica \\ Politecnico di Torino \\ 10129 Torino, Italy \\ emanuele.viterbo@polito.it
}

\begin{abstract}
We study the error probability performance of rotated lattice constellations in frequency-flat block-fading channels. In particular, we use the sphere lower bound on the underlying infinite lattice as a performance benchmark. We show that the sphere lower bound has full diversity. We observe that optimally rotated lattices with largest known minimum product distance perform very close to the lower bound, while the ensemble of random rotations is shown to lack diversity and perform far from it. We furthermore use the bound in the multiple-antenna case, and we observe that the Golden code, the optimal full-rate full-diversity $2 \times 2$ space-time block code, is also very close to the lower bound.
\end{abstract}

\section{INTRODUCTION}

In this paper, we study the family of full rate multidimensional signal constellations carved from lattices in frequencyflat fading channels with $N$ degrees of freedom. In particular, we consider the uncoded case, i.e., no time redundancy is added to the transmitted signal. Current best constellations are designed to achieve full diversity and maximize the minimum product distance [1], [2], [3]. Unfortunately, to date, there exists no benchmark to compare the performance of rotated lattice constellations. Recent reference [4] gives an approximation to the error probability of multidimensional constellations in fading channels. Unfortunately, the approximation is not tight and does not have full diversity.

In this paper, we use the sphere lower bound ${ }^{2}$ (SLB), as a benchmark for the performance of such uncoded lattice constellations. The SLB dates back to Shannon's work [6], and gives a lower bound to the error probability of spherical codes with a given length in the additive white Gaussian noise (AWGN) channel. The application of the SLB to infinite lattices and lattice codes was studied in [7], [8] for the AWGN channel. This SLB yields a lower bound to the error probability of infinite lattices regardless of the lattice structure. An approximated SLB was derived in [9] for spherical codes over the Rayleigh fading channel. Fozunbal et al. [10] extended the SLB to coded communication over the multipleantenna block-fading channel. A remarkable result of [10] is

\footnotetext{
${ }^{1}$ The work by A. Guillén i Fàbregas has been supported by the Australian Research Council (ARC) Grants DP0344856 and DP0558861, and by the University of South Australia Australian Competitive Grant Development Scheme. The work by E. Viterbo has been supported by the International Visiting Researcher Program of the University of South Australia and the by the STREP project No. IST-026905 (MASCOT) within the sixth framework programme of the European Commission.

${ }^{2}$ Literature commonly refers to such bound as sphere-packing bound. In order to avoid possible confusion with lattice terminology, we will refer to it as sphere lower bound, since its computation is not based on the packing radius of the lattice [5].
}

that, for a fixed number of antennas and blocks, as the code length grows, the SLB converges to the outage probability of the channel with Gaussian inputs [11]. Unfortunately, the outage probability [12], [11] and the SLB of [10] are very far from the actual error probability of uncoded multidimensional constellations. Moreover, as the block length increases, the performance of uncoded modulations degrades, and therefore, the outage probability and the SLB of [10] are not very useful as performance benchmarks.

In this paper, we use the SLB of the infinite lattice as a benchmark for comparing multidimensional constellations in the block-fading channel with single and multiple antennas. We first show that the SLB of infinite lattice rotations for the block-fading channel has full diversity regardless of the block length. We illustrate that as the block length increases, the SLB increases as well. We also show that multidimensional constellations obtained by algebraic rotations with largest minimum product distance obtained from pairwise error probability criteria [1], [2], [3] perform very close to the lower bound and that the ensemble of random rotations does not achieve full diversity. We furthermore use the SLB in $N \times N$ multipleinput multiple-output (MIMO) channels, in order to assess the performance of full-rate full-diversity algebraic space-time block codes (STBC) [13], [14], [15]. In particular we observe that the Golden code [14], the optimal full-rate full-diversity $2 \times 2$ space-time block code, is very close to the bound.

\section{SYSTEM MODEL}

We first consider a single-antenna flat fading channel whose discrete-time received signal vector is given by

$$
\mathbf{y}_{\ell}=\mathbf{H} \mathbf{x}_{\ell}+\mathbf{z}_{\ell}, \quad \ell=1, \cdots, L
$$

where $\mathbf{y}_{\ell} \in \mathbb{R}^{N}$ is the $N$-dimensional real received signal vector, $\mathbf{x}_{\ell} \in \mathbb{R}^{N}$ is the $N$-dimensional real transmitted signal vector, $\mathbf{H}=\operatorname{diag}(\mathbf{h}) \in \mathbb{R}^{N \times N}$, with $\mathbf{h}=\left(h_{1}, \ldots, h_{N}\right) \in$ $\mathbf{R}^{N}$, is the flat fading diagonal matrix, and $\mathbf{z} \in \mathbb{R}^{N}$ is the Gaussian noise vector with pdf

$$
p(\mathbf{z})=\left(2 \pi \sigma^{2}\right)^{-\frac{N}{2}} \exp \left(-\frac{\|\mathbf{z}\|^{2}}{2 \sigma^{2}}\right)
$$

A frame is composed of $L, N$-dimensional modulation symbols or of $N L$ one-dimensional channel uses. The case of complex signals obtained from 2 orthogonal real signals can be similarly modeled by (1) by replacing $L$ with $L^{\prime}=2 L$.

We assume that the fading matrix $\mathbf{H}$ is constant during one frame and it changes independently from frame to frame. 


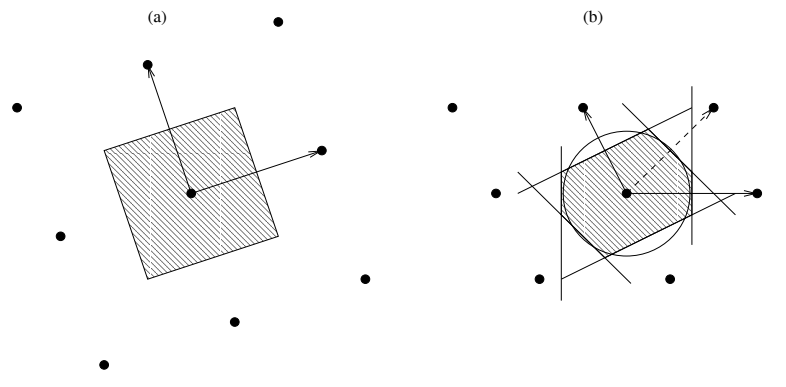

Fig. 1. The decision regions of the rotated $\mathbf{Z}^{2}$ lattice: (a) before fading, (b) after fading $\mathbf{h}=(1,0.5)$.

This corresponds to the block-fading channel with $N$ blocks [12]. We further assume perfect channel state information (CSI) at the receiver, i.e., the receiver perfectly knows the fading coefficients. Therefore, for a given fading realization, the channel transition probabilities are given by

$$
p(\mathbf{y} \mid \mathbf{x}, \mathbf{H})=\left(2 \pi \sigma^{2}\right)^{-\frac{N}{2}} \exp \left(-\frac{1}{2 \sigma^{2}}\|\mathbf{y}-\mathbf{H x}\|^{2}\right)
$$

Moreover, we assume that the real fading coefficients are Rayleigh-distributed. For future use, we define the coefficients $\gamma_{n}=h_{n}^{2}$ for $n=1, \ldots, N$, which correspond to the fading power gains with distribution

$$
p(\gamma)=e^{-\sum_{n=1}^{N} \gamma_{n}} .
$$

Finally, we define the signal-to-noise ratio (SNR) as $\rho=1 / \sigma^{2}$.

\section{A. Multidimensional Lattice Constellations}

We assume that the transmitted signal vectors $\mathrm{x}$ belong to an $N$-dimensional signal constellation $\mathcal{S} \subseteq \mathbb{R}^{N}$. We consider signal constellations $\mathcal{S}$ that are generated as a finite subset of points of the infinite lattice $\Lambda=\left\{\mathbf{M u}: \mathbf{u} \in \mathbb{Z}^{N}\right\}$ with full rank generator matrix $\mathbf{M} \in \mathbb{R}^{N \times N}$ [5]. For normalization purposes we fix $\operatorname{det}(\mathbf{M})=1$. For a given channel realization, we define the faded lattice as the lattice $\Lambda^{\prime}=\left\{\mathbf{M}^{\prime} \mathbf{u}: \mathbf{u} \in\right.$ $\left.\mathbb{Z}^{N}\right\}$, whose generator matrix is given by $\mathbf{M}^{\prime}=\mathbf{H M}$.

In order to simplify the labeling operation, constellations are of the type $\mathcal{S}=\left\{\mathbf{M u}+\mathbf{x}_{0}: \mathbf{u} \in \mathbb{Z}_{m}^{N}\right\}$, where $\mathbb{Z}_{m}=$ $\{0,1, \ldots, m-1\}=(m-\mathrm{PAM})^{\mathrm{N}}, \log _{2}(m)$ is the number of bits per dimension and $\mathbf{x}_{0}$ is an offset vector which minimizes the average transmitted energy. Therefore, the rate of such constellations is $R=\log _{2} m$ bit/dimension. This is usually referred to as full-rate uncoded transmission.

In order to avoid shaping loss it is convenient to use cubic lattice constellations, so that $\mathbf{M}$ should be an orthogonal matrix $\left(\mathbf{M M}^{T}=\mathbf{I}\right)$ [1], [2]. Nevertheless, this will not be required in the calculation of the SLB.

\section{B. Maximum Likelihood Decoding Error Probability}

At a given $\ell$, a maximum likelihood (ML) decoder with perfect CSI makes an error whenever

$$
\left\|\mathbf{y}_{\ell}-\mathbf{H w}\right\|^{2} \leq\left\|\mathbf{y}_{\ell}-\mathbf{H} \mathbf{x}\right\|^{2}
$$

for some $\mathbf{w} \in \mathcal{S}, \mathbf{w} \neq \mathbf{x}$. These inequalities define the so called decision region around $\mathbf{x}$ (see Figure 1). Under ML decoding, the frame error probability is then given by

$$
P_{\mathrm{f}}(\rho)=\mathbb{E}\left[P_{\mathrm{f}}(\rho \mid \mathbf{h})\right]=\mathbb{E}\left[1-\left(1-P_{\mathrm{s}}(\rho \mid \mathbf{h})\right)^{L}\right]
$$

where $P_{\mathrm{f}}(\rho \mid \mathbf{h})$ and $P_{\mathrm{s}}(\rho \mid \mathbf{h})$ are the frame and $N$-dimensional symbol error probabilities for a given channel realization and the average is taken over the fading distribution. For a given constellation $\mathcal{S}$, we can write that

$$
P_{\mathrm{S}}(\rho \mid \mathbf{h})=\mathbb{E}\left[P_{\mathrm{S}}(\rho \mid \mathbf{x}, \mathbf{h})\right]=\frac{1}{|\mathcal{S}|} \sum_{\mathbf{x} \in \mathcal{S}} \int_{\mathbf{y} \notin \mathcal{V}(\mathbf{x}, \mathbf{h})} p(\mathbf{y} \mid \mathbf{x}, \mathbf{h}) d \mathbf{y}
$$

where $\mathcal{V}(\mathbf{x}, \mathbf{h})$ is the decision region or Voronoi region for a given multidimensional lattice constellation point $\mathrm{x}$ and fading $\mathbf{H}$ (see Figure 1). Computing the regions $\mathcal{V}(\mathbf{x}, \mathbf{h})$ and the exact error probability is in general a very hard problem [7]. In this paper we use the SLB [6] as a lower bound on $P_{\mathrm{f}}$.

We define the diversity order as the asymptotic (for large SNR) slope of $P_{\mathrm{f}}$ versus SNR in a log-log scale, namely,

$$
d=-\lim _{\rho \rightarrow \infty} \frac{\log P_{\mathrm{f}}(\rho)}{\log \rho} .
$$

In words, the diversity order is the maximum number of deep fades that a signal constellation can support, i.e., the ML decoder will decode correctly in presence of $d-1$ deep fades. We say that a constellation $\mathcal{S}$ has full diversity if $d=N$.

\section{Sphere Lower Bound of A FAded LATtice}

In this section, we recall the basics of the SLB for infinite lattices $\mathcal{S}=\Lambda$ [7], [8] and we apply it to bound $P_{\mathrm{f}}(\rho)$.

The first simplification stems from the geometrical uniformity of lattices [7], [8], i.e.,

$$
\mathcal{V}(\mathbf{x}, \mathbf{h})=\mathcal{V}(\mathbf{w}, \mathbf{h}) \quad \forall \mathbf{x}, \mathbf{w} \in \Lambda
$$

namely, for a given fading realization, the Voronoi regions of all lattice points are equal. Let $\mathcal{V}_{\Lambda}(\mathbf{h})$ denote such Voronoi region of the faded lattice. Therefore, and without loss of generality, we safely assume the transmission of the all-zero codeword, i.e., $\mathbf{x}_{\ell}=\mathbf{0}, \ell=1, \ldots, L$. Then, the error probability is given by

$$
P_{\mathrm{f}}(\rho)=1-\mathbb{E}\left[\left(1-\int_{\mathbf{z} \notin \mathcal{V}_{\Lambda}(\mathbf{h})} p(\mathbf{z}) d \mathbf{z}\right)^{L}\right] .
$$

Due to the circular symmetry of the Gaussian noise, replacing $\mathcal{V}_{\Lambda}(\mathbf{h})$ by an $N$-dimensional sphere $\mathcal{B}(\mathbf{h})$ (see Figure 1 ) of radius $R(\mathbf{h})$ with the same volume [6], yields the corresponding SLB on the lattice performance [7], [8]

$$
P_{\mathrm{f}}(\rho) \geq P_{\mathrm{slb}}(\rho) \triangleq 1-\mathbb{E}\left[\left(1-\int_{\mathbf{z} \notin \mathcal{B}(\mathbf{h})} p(\mathbf{z}) d \mathbf{z}\right)^{L}\right]
$$

Since the volume of $\mathcal{B}(\mathbf{h})$ is [5]

$$
\operatorname{vol}(\mathcal{B}(\mathbf{h}))=\frac{\pi^{\frac{N}{2}} R(\mathbf{h})^{N}}{\Gamma\left(\frac{N}{2}+1\right)}
$$

with

$$
\Gamma(x) \triangleq \int_{0}^{\infty} t^{x-1} e^{-t} d t
$$

being the Gamma function [16]. Equating (7) to the fundamental volume of the lattice (volume of the Voronoi region) given by

$$
\operatorname{vol}\left(\mathcal{V}_{\Lambda}(\mathbf{h})\right)=\operatorname{det}(\mathbf{H M})=\prod_{n=1}^{N} h_{n}
$$


we obtain the sphere radius

$$
R(\mathbf{h})^{2}=\frac{1}{\pi} \Gamma\left(\frac{N}{2}+1\right)^{\frac{2}{N}}\left(\prod_{n=1}^{N} \gamma_{n}\right)^{1 / N}
$$

The probability that the noise brings the received point outside the sphere in (6) is simply expressed as [6], [7], [8]

$$
P_{\mathrm{slb}}(\rho)=1-\mathbb{E}\left[\left(1-\bar{\Gamma}\left(\frac{N}{2}, \frac{R(\mathbf{h})^{2}}{2} \rho\right)\right)^{L}\right]
$$

where

$$
\bar{\Gamma}(a, x)=\frac{1}{\Gamma(a)} \int_{x}^{\infty} t^{a-1} e^{-t} d t
$$

is the incomplete gamma function [16].

We are now ready for the following result:

Theorem 1: In a single-antenna block-fading channel with $N$ fading blocks, the sphere lower bound on error probability given in (10) has full diversity for any $L \geq 1$, i.e.,

$$
d=-\lim _{\rho \rightarrow \infty} \frac{\log P_{\mathrm{slb}}(\rho)}{\log \rho}=N .
$$

Proof: The proof is omitted for the sake of space limitation.

The previous theorem asserts that the best lattice in a channel with $N$ fading blocks cannot have diversity larger than $N$. This result is non-trivial, and very important for constellation design. Pairwise error probability analysis yields that full diversity lattices can achieve full diversity [1], [2], [3], but no converse based on the lattice structure has been proved so far for any $L$. Clearly, if we construct our signal constellation $\mathcal{S}$ as a subset of points of an $N$-dimensional lattice, $\mathcal{S}$ cannot have diversity larger than $N$ either.

In order to evaluate (10), we need to perform a multidimensional numerical integral over the joint distribution $p(\gamma)$. However, by carefully observing the expression of $R(\mathbf{h})^{2}$, we can see that we only need to know the pdf of the product of fading coefficients. It is not difficult to show that the characteristic function of the random variable

$$
\zeta=\log \prod_{n=1}^{N} \gamma_{n}=\sum_{n=1}^{N} \log \gamma_{n}
$$

is given by

$$
G_{\zeta}(f)=\Gamma(1-j 2 \pi f)^{N}
$$

For $N>1$, unfortunately, the closed form inverse transform of this function is not available, but we can nevertheless compute the pdf $p_{\zeta}(z)$ numerically by using an inverse fast Fourier transform (FFT). As an example, Figure 2 shows the SLB for $N=2,4,8,16,32,64$ and $L=1$. As anticipated by Theorem 1 , the curves get steeper as $N$ increases. Moreover, Figure 3 shows the SLB for $N=2,4$ and $L=1,10,100,1000$. For a given $N$, all curves have the same diversity. We note that the SNR $\rho=1 / \sigma^{2}$ is relative to the infinite lattice with $\operatorname{vol}(\Lambda)=$ 1 , since average transmitted energy cannot be defined.

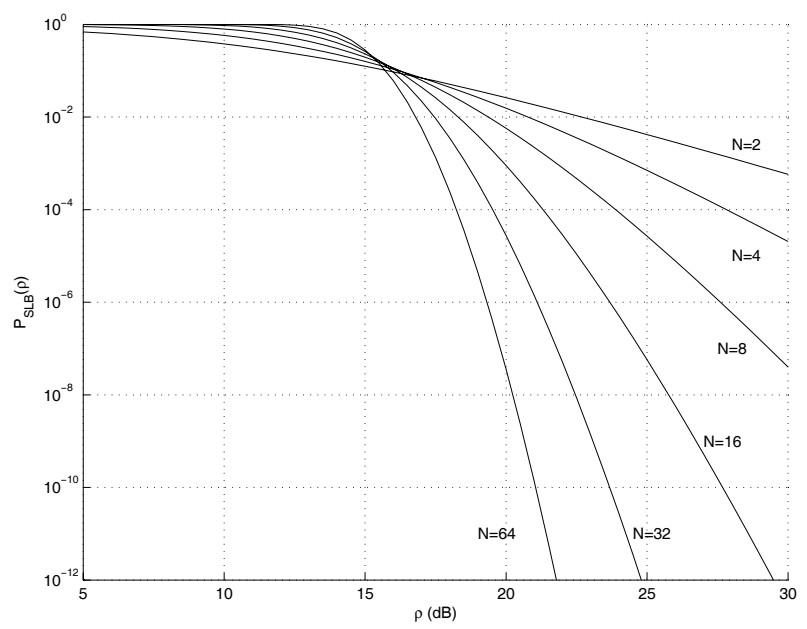

Fig. 2. Sphere lower bound $P_{\mathrm{slb}}(\rho)$ for $N=2,4,8,16,32,64$ and $L=1$.

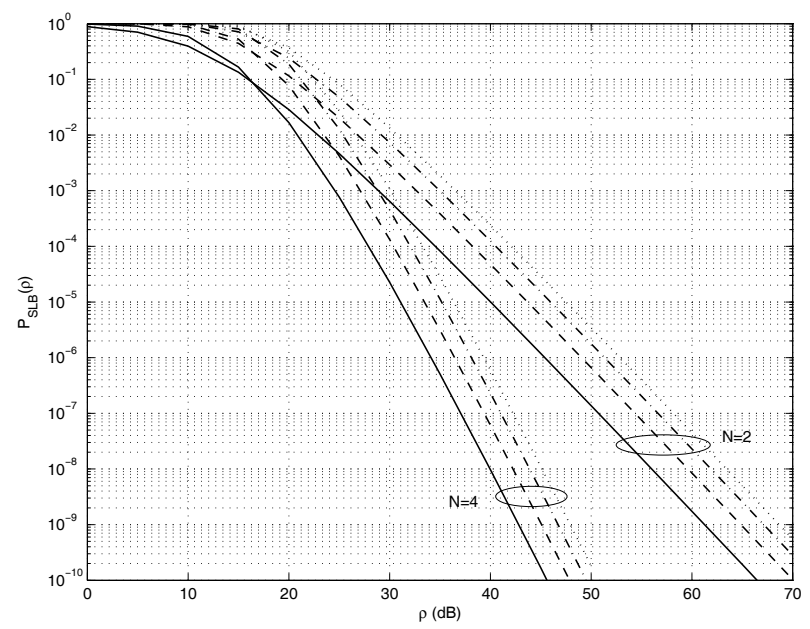

Fig. 3. Sphere lower bound $P_{\mathrm{slb}}(\rho)$ for $N=2,4$ and $L=1$ (continuous), $L=10$ (dashed), $L=100$ (dashed-dotted) and $L=1000$ (dotted).

\section{PERFormance of Rotated LATtices}

In this section, we give a number of examples that use the SLB as a benchmark for comparing some lattices obtained by algebraic rotations, as explained in section II-A. In particular, we will use the best known or optimal algebraically rotated $\mathbb{Z}^{N}$ lattices in terms of largest minimum product distance [1], [2], [17], [3]. As we shall see, these rotations perform very close to the lower bound. Furthermore, we will show that the ensemble of random rotations does not have full diversity. Hence, specific constructions that guarantee full diversity and largest minimum product distance are very important.

To illustrate this, Figures 4 and 5 compare the frame error probability $P_{\mathrm{f}}(\rho)$ of some rotations (obtained by simulation of the infinite lattice using a Schnorr-Euchner decoder [18]) with the $P_{\mathrm{slb}}(\rho)$ for $N=2,4$ and $L=1,100$. We compare the optimal rotations with largest minimum product distance of dimension $N=2,4$, namely, the cyclotomic rotation of dimension $N=2$ and the Krüskemper rotation for $N=4$ (see [1], [2], [17] for more information on these constructions). The corresponding rotation matrices are also available in [3].

We compare by simulation the performance of these full- 
diversity algebraic rotations with the average performance of the ensemble of random rotations. At every frame, we generate a random matrix $\mathbf{A}$ with zero mean and unit variance i.i.d. Gaussian entries. We then performe a $\mathbf{A}=\mathbf{Q R}$ decomposition and let $\mathbf{M}=\mathbf{Q}$. This is the simplest way of generating the ensemble of random rotations (orthogonal matrices) with the Haar distribution [19]. As we observe, algebraic rotations perform very close to $P_{\mathrm{slb}}(\rho)$. On the other hand, the average error probability over the ensemble of random rotations, lacks of the full diversity and exhibits bad performance.

As $N$ increases, algebraic rotations with largest minimum product distance show some gap to $P_{\mathrm{slb}}(\rho)$. This is due to the fact that for large $N$, the minimum product distance is not the only relevant design parameter for optimizing the coding gain.

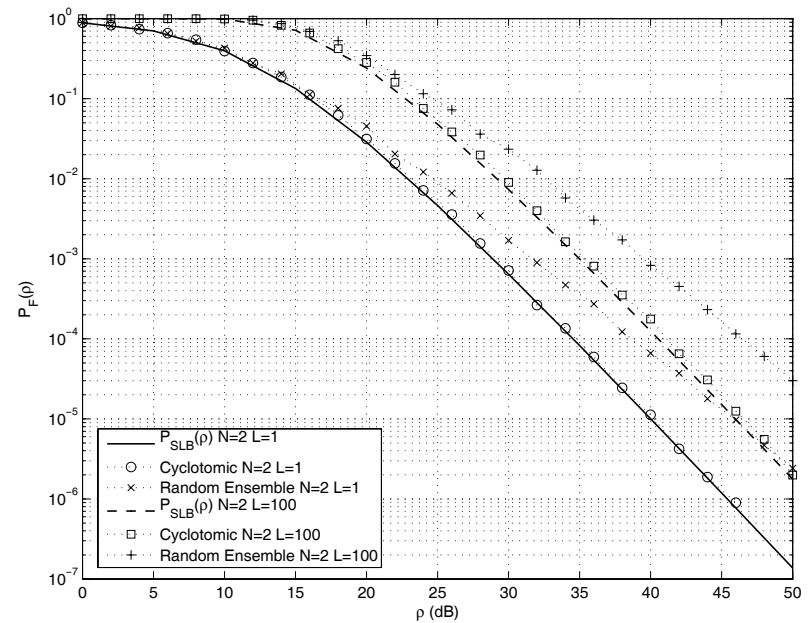

Fig. 4. Frame error probability $P_{\mathrm{f}}(\rho)$ and sphere lower bound $P_{\mathrm{slb}}(\rho)$ for $N=2$ and $L=1,100$.

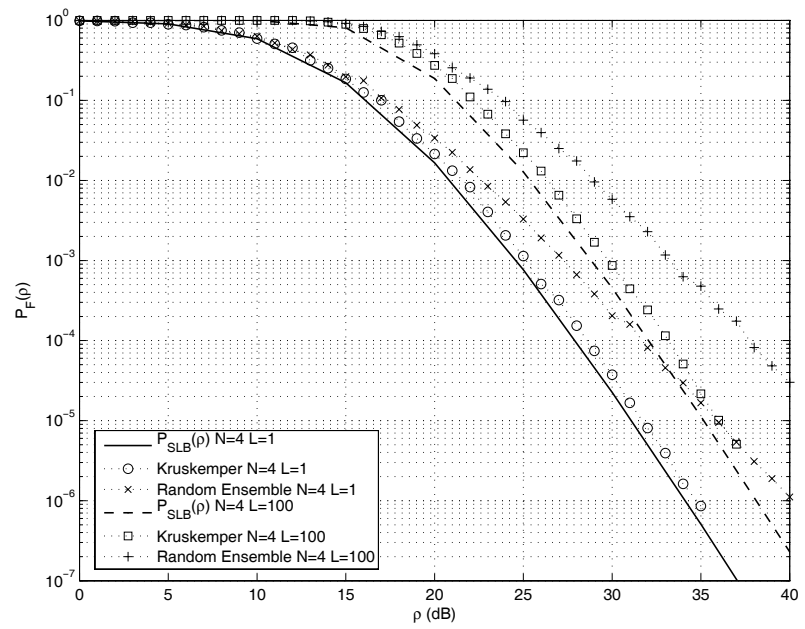

Fig. 5. Frame error probability $P_{\mathrm{f}}(\rho)$ and sphere lower bound $P_{\mathrm{slb}}(\rho)$ for $N=4$ and $L=1,100$.

\section{PERformance of MUltidimensional Signal SETS}

So far, we have seen that the performance of algebraic rotations is very close to the lower bound $P_{\mathrm{slb}}(\rho)$. In practical communications systems, we shall use finite signal alphabets and the performance of the infinite rotated lattice should serve mainly as a guideline. Unfortunately, we do not have a bound similar to $P_{\mathrm{slb}}(\rho)$ for the finite case to take into account the boundary effects. We have the following conjecture regarding the performance of multidimensional finite signal sets.

Conjecture 1: The best multidimensional signal set using $m$-PAM is the one whose performance of the infinite rotated lattice with the same generator matrix $\mathbf{M}$ is closest to $P_{\mathrm{slb}}(\rho)$.

As we shall see in the following example, as $m$ increases, the performance of the multidimensional signal constellation approaches that of the infinite rotated lattice, despite the boundary effects. This is precisely the continuity argument used in [8] for lattice codes. Indeed, Figures 6 and 7 show the performance for $N=2,4$ and $L=1,100$ of the signal constellations obtained from $m$-PAM with the optimal algebraic rotation. In the comparison with the infinite lattice (circles) and $P_{\mathrm{slb}}(\rho)$, we observe all curves are within $1.5 \mathrm{~dB}$.

Note that the SNR axis does not take into account the different average energies of the finite constellations and that we assume that the minimum distance of the $m$-PAM is 1 for comparison to the infinite lattice lower bound. In order to plot the performance in terms of $\frac{E_{b}}{N_{0}}=\frac{E_{b}}{2} \rho$ it is enough to shift the curves by

$$
10 \log _{10}\left(\frac{m^{2}-1}{24 \log _{2} m}\right)
$$

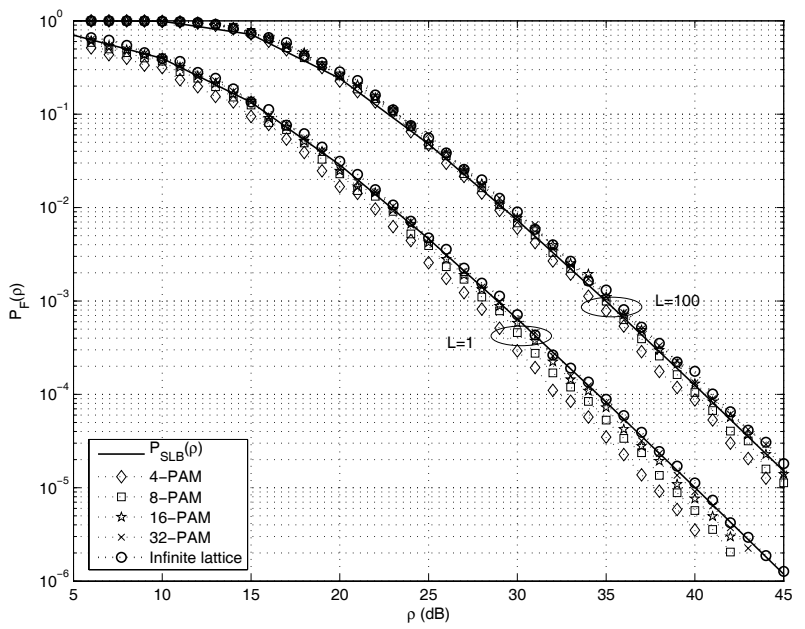

Fig. 6. Frame error probability $P_{\mathrm{f}}(\rho)$ of the finite constellation generated with $4,8,16,32$-PAM, $P_{\mathrm{f}}(\rho)$ of the infinite lattice and sphere lower bound $P_{\text {slb }}(\rho)$ for $N=2$ and $L=1,100$ with the cyclotomic rotation.

\section{ApPliCATION to $N \times N$ MiMO ChanNELS}

In this section we show how the SLB can be applied to give a lower bound on the performance of algebraic STBCs [13], [14], [15] over $N \times N$ MIMO fading channels. Due to their lattice structure, these STBC constructions are based on a rotated real $\mathbb{Z}^{2 N^{2}}$ lattice, and admit an equivalent vectorized real channel model [13], [14], [15],

$$
\mathbf{y}_{\ell}=\widetilde{\mathbf{H}} \mathbf{M} \mathbf{x}_{\ell}+\mathbf{z}_{\ell} \quad \ell=1, \cdots, L
$$

where $\mathbf{y}_{\ell}, \mathbf{x}_{\ell}, \mathbf{z}_{\ell} \in \mathbb{R}^{2 N^{2}}$ and $\widetilde{\mathbf{H}}, \mathbf{M} \in \mathbb{R}^{2 N^{2} \times 2 N^{2}}$ are obtained from the complex channel matrix $\mathbf{H} \in \mathbb{C}^{N \times N}$ and the specific code construction [13], [14], [15]. Model (13) is used at the decoding side with a $2 N^{2}$-dimensional real lattice decoder. 


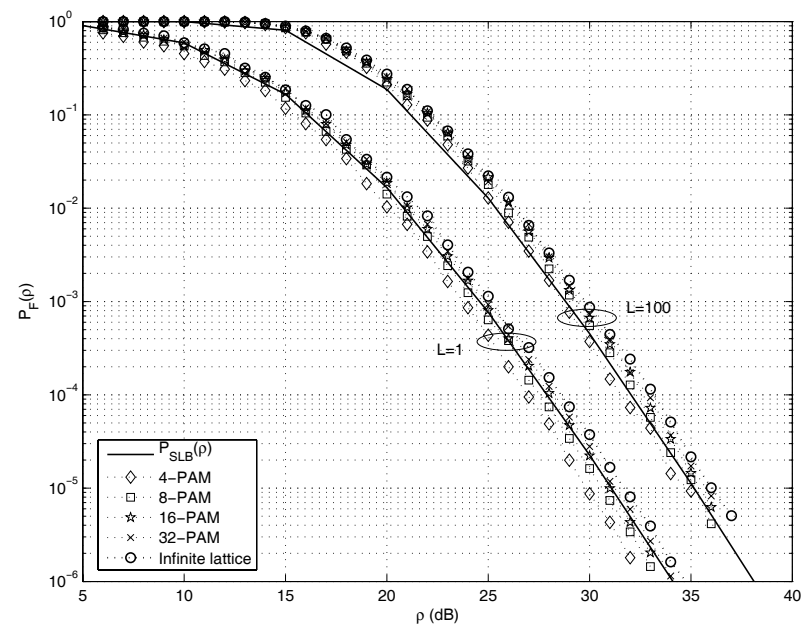

Fig. 7. Frame error probability $P_{\mathrm{f}}(\rho)$ of the finite constellation generated with $4,8,16,32$-PAM, $P_{\mathrm{f}}(\rho)$ of the infinite lattice and sphere lower bound $P_{\mathrm{slb}}(\rho)$ for $N=4$ and $L=1,100$ with the Krüskemper rotation.

Therefore, the SLB can be directly applied in the MIMO case by replacing $N$ by $2 N^{2}$ in (10) and by letting

$$
\operatorname{vol}\left(\mathcal{V}_{\Lambda}(\mathbf{H})\right)=\operatorname{det}(\widetilde{\mathbf{H}})=|\operatorname{det}(\mathbf{H})|^{2 N}
$$

We have the following

Theorem 2: In a $N \times N$ MIMO channel the sphere lower bound on the error probability has full diversity for any $L \geq 1$, i.e.,

$$
d=-\lim _{\rho \rightarrow \infty} \frac{\log P_{\mathrm{slb}}(\rho)}{\log \rho}=N^{2} .
$$

In the following, we illustrate with an example the SLB and simulated performance of the Golden code [14]. The matrices $\widetilde{\mathbf{H}}$ and $\mathbf{R}$ for the Golden code are given in [3]. The Golden code is the optimal full-rate full-diversity $2 \times 2$ algebraic STBC. The Golden code has the largest minimum non-vanishing determinant [14] and therefore achieves the diversity-multiplexing tradeoff of [20]. Figure 8 shows the SLB $P_{\mathrm{slb}}(\rho)$ and the Golden code simulation in a $2 \times 2$ MIMO with $L=1,100$. Once again, we observe that the simulation is very close to the bound.

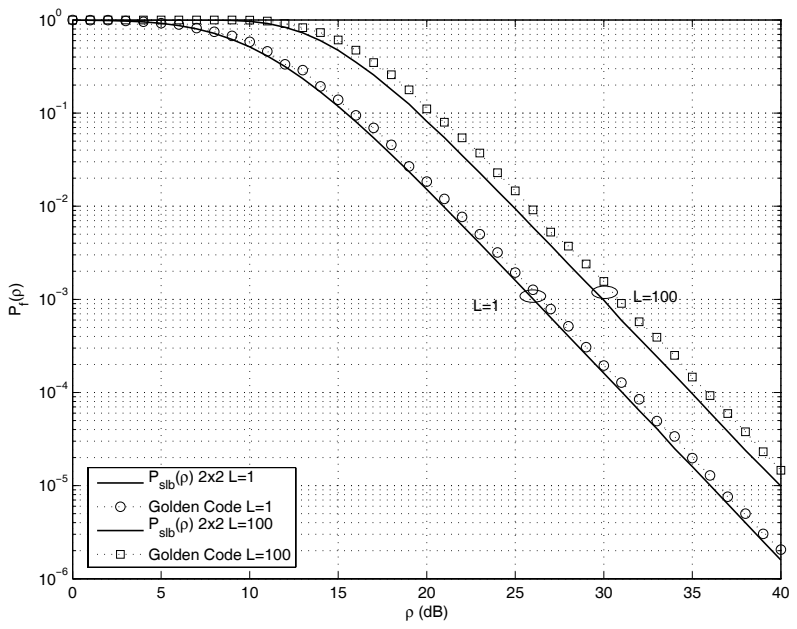

Fig. 8. Frame error probability $P_{\mathrm{f}}(\rho)$ of the infinite lattice generated by the Golden code and sphere lower bound $P_{\text {slb }}(\rho)$ for $L=1,100$ in a $2 \times 2$ multiple antenna channel.

\section{CONCLUSIONS}

In this paper we have studied the performance of multidimensional rotated lattice constellations. We have applied the sphere lower bound for the infinite lattice to the block-fading channel and proved that the bound has full diversity. We have shown that optimally rotated algebraic lattices perform very close to the bound, while the average over the ensemble of random rotations does not. Furthermore, we have shown that finite constellations obtained from the rotation of $\{m-\mathrm{PAM}\}^{N}$ constellations perform close to the bound as $m$ gets large. We have conjectured that optimal multidimensional signal sets with $m$-PAM constellation are obtained from rotated lattices whose performance is closest to the sphere lower bound. We have finally shown the applicability of the above results to the multiple antenna case.

\section{REFERENCES}

[1] E. Bayer-Fluckiger, F. Oggier, E. Viterbo, "New algebraic constructions of rotated $\mathbb{Z}^{n}$-lattice constellations for the Rayleigh fading channel," IEEE Trans. on Inf. Theory, vol. 50, no. 4, pp. 702-714, Apr. 2004.

[2] F. Oggier and E. Viterbo, "Algebraic number theory and code design for rayleigh fading channels," Foundations and Trends in Communications and Information Theory, vol. 1, pp. 333-415, 2004.

[3] E. Viterbo and F. Oggier, "Tables of algebraic rotations and The Golden code homepage," http://www.tlc.polito.it/ viterbo.

[4] J.-C. Belfiore and E. Viterbo, "Approximating the error probability for the independent Rayleigh fading channel," in 2005 Int. Symp. on Inf. Theory, Adelaide, Australia, Sept. 2005.

[5] J. H. Conway and N. J. A. Sloane, Sphere packings, lattices and groups, Springer, 3rd edition, 1999.

[6] C. E. Shannon, "Probability of error for optimal codes in a gaussian channel," The Bell System Technical Journal, vol. 38, no. 3, pp. 279324, May 1959.

[7] E. Viterbo and E. Biglieri, "Computing the Voronoi cell of a lattice: The diamond-cutting algorithm," IEEE Trans. on Inf. Theory, vol. 42, no. 1, pp. 161-171, Jan. 1996.

[8] V. Tarokh, A. Vardy and K. Zeger, "Universal bound on the performance of lattice codes," IEEE Trans. on Inf. Theory, vol. 45, no. 2, pp. 670-681, Mar. 1999.

[9] S. Vialle and J. Boutros, "Performance of optimal codes on Gaussian and Rayleigh fading channels: a geometrical approach," in 37th Allerton Conf. on Commun., Control and Comput., Monticello, IL., Sept. 1999.

[10] M. Fozunbal, S. W. McLaughlin and R. W. Schafer, "On performance limits of space-time codes: a sphere-packing bound approach," IEEE Trans. on Inf. Theory, vol. 49, no. 10, pp. 2681-2687, Oct. 2003.

[11] I. E. Telatar, "Capacity of multi-antenna Gaussian channels," European Trans. on Telecomm., vol. 10, no. 6, pp. 585-596, November 1999.

[12] L. H. Ozarow, S. Shamai and A. D. Wyner, "Information theoretic considerations for cellular mobile radio," IEEE Trans. on Vehicular Tech., vol. 43, no. 2, pp. 359-378, May 1994.

[13] H. El Gamal and M. O. Damen, "Universal space-time coding," IEEE Trans. on Inf. Theory, vol. 48, no. 5, pp. 1097-1119, May 2003.

[14] J.-C. Belfiore, G. Rekaya and E. Viterbo, "The Golden code: a $2 \times 2$ full-rate space-time code with nonvanishing determinants," IEEE Trans. on Inf. Theory, vol. 51, no. 4, pp. 1432-1436, Apr. 2005.

[15] F. Oggier, G. Rekaya, J.-C. Belfiore, and E. Viterbo, "Perfect space-time block codes," accepted to IEEE Trans. on Inf. Theory.

[16] M. Abramowitz and I. A. Stegun, Handbook of Mathematical Functions with Formulas, Graphs and Mathematical Tables, New York: Dover Press, 1972.

[17] F. Oggier, Algebraic methods for channel coding, Ph.D. thesis, Ecole Polytechnique Fédérale de Lausanne (EPFL), 2005.

[18] E. Agrell, T. Eriksson, A. Vardy and K. Zeger, "Closest point search in lattices," IEEE Trans. on Inf. Theory, vol. 48, pp. 2201-2214, Aug. 2002.

[19] G. W. Stewart, "The efficient generation of random orthogonal matrices with an application to condition estimation," SIAM J. Numer. Anal., vol. 17, pp. 403-409, 1980.

[20] L. Zheng and D. Tse, "Diversity and multiplexing: A fundamental tradeoff in multiple antenna channels," IEEE Trans. on Inf. Theory, vol. 5, no. 49, pp. 1073-1096, May 2003. 